\title{
Seroprevalencia de infección por Leptospira en habitantes de barrios periféricos de Cali
}

\author{
Beatriz Eugenia Ferro, Ana Lucía Rodríguez, Mauricio Pérez, Bruno Luis Travi \\ Centro Internacional de Entrenamiento e Investigaciones Médicas, CIDEIM, Cali, Colombia.
}

Introducción. La leptospirosis es una enfermedad zoonótica urbana y rural que afecta a los humanos en todos los continentes, aunque su transmisión y presentación clínica puede variar. En Colombia hay poca información acerca de la importancia de la leptospirosis en entornos urbanos.

Objetivos. Establecer la seroprevalencia de infección por Leptospira en humanos, identificar la reactividad serológica y explorar factores asociados con la seropositividad.

Materiales y métodos. Se realizó un estudio transversal en 259 habitantes de barrios periféricos de Cali, estratificado por sexo y edad. Se obtuvo información general de cada persona, tiempo de residencia en el barrio, ocupación, exposición a fuentes contaminadas como agua o animales, y suero para la prueba de microaglutinación con 19 serovares de Leptospira.

Resultados. La población evaluada correspondió principalmente a estudiantes (37,1\%), amas de casa (32\%) y trabajadores en oficios varios (24,6\%). Se encontraron anticuerpos antiLeptospira en 23,3\% (IC95\% 18,3 a 28,3) de las personas, siendo más frecuente en mayores de 57 años y significativamente mayor en hombres que en mujeres $(p=0,045)$. Se encontró asociación entre la seropositividad y el contacto con animales $(p=0,038)$. De los 19 serovares evaluados, se detectó reactividad frente a 16, pero los títulos de anticuerpos fueron bajos. Conclusiones. La alta prevalencia de infección encontrada en el estudio sugiere la transmisión frecuente de leptospiras en barrios de Cali considerados de alto riesgo. Se destaca el contacto con animales como un factor asociado con la presencia de anticuerpos contra leptospiras.

Palabras clave: estudios seroepidemiológicos, leptospirosis, anticuerpos, Colombia.

Seroprevalence of Leptospira infection in habitants of peripheral neighborhoods in Cali, Colombia

Introduction. Leptospirosis is a zoonotic disease with varying modes of transmission and clinical expression that affects humans inhabiting urban and rural areas worldwide. In Colombia, little is known regarding leptospirosis in urban settings.

Objectives. To establish the seroprevalence of leptospiral infection in humans, to assess the serological reactivity and to identify factors associated with seropositivity.

Materials and methods. We conducted an infection prevalence study in 259 inhabitants of peripheral neighborhoods in the city of Cali, Colombia, stratified by sex and age. Sociodemographic information, time residing in the same neighborhood, occupation, exposure to potentially infectious sources like water or animals, and serum used in a Microagglutination test against 19 Leptospira serovars were obtained.

Results. The population was composed mainly of students (37.1\%), housewives (32\%) and workers in non-formal activities (24.6\%). The prevalence of anti-Leptospira antibodies was $23.3 \%$ (CI95\% 18.3-28.3), with high frequency in people older than 57 years of age and significantly higher in men than women $(p=0.045)$. There was an association between the presence of antibodies and contact with animals $(p=0.038)$. Reactivity was observed against 16 of the 19 serovars evaluated, but the antibody titers were low.

Conclusions. The high infection seroprevalence found in this study suggests frequent contact with Leptospira in putatively high risk neighborhoods of Cali. Contact with animals was the most significant factor associated with the presence of anti-Leptospira antibodies.

Keywords: seroepidemiologic studies, leptospirosis, antibodies, Colombia. 
La leptospirosis es una enfermedad zoonótica de importancia mundial (1) que afecta a los humanos en todos los continentes, tanto en contextos urbanos como rurales y en climas templados y tropicales (2).Tradicionalmente la leptospirosis se ha considerado una enfermedad ocupacional o que esporádicamente ocurre en zonas rurales (1-3), pero actualmente, debido a brotes en zonas urbanas y en poblaciones con diferentes niveles de riesgo, se la considera como una de las enfermedades infecciosas reemergentes (1,3-5).

La presentación clínica de la enfermedad en humanos varía desde cuadros subclínicos hasta la enfermedad fulminante (6). Este amplio espectro de síntomas hace que el diagnóstico de la leptospirosis sea difícil (2), siendo necesario el uso combinado de pruebas diagnósticas y criterios clínicos y epidemiológicos.

La infección en los seres humanos ocurre directamente a través del contacto de las mucosas con orina de animales infectados o indirectamente a través del contacto con agua o suelos contaminados $(1,7,8)$. Los animales infectados pueden ser hospederos de mantenimiento o accidentales $\mathrm{y}$, a su vez, portar y transmitir diferentes serovares de Leptospira (5). La prevalencia de diferentes serovares en una población humana depende del hospedero presente, de los serovares que este hospedero porte y también de las condiciones sanitarias locales, la ocupación y las prácticas de la población (1). De acuerdo con lo anterior, la transmisión y la expresión clínica de la leptospirosis puede variar según los contextos medioambientales y socioeconómicos (6). El estudio de cada contexto, así como la vigilancia continua, son necesarios para determinar la magnitud del problema y las características epidemiológicas de la leptospirosis que permitan el desarrollo e implementación de medidas de control efectivas (2).

\footnotetext{
Correspondencia:

Beatriz Eugenia Ferro, CIDEIM, Avenida 1 Norte № 3-03, Cali, Colombia.

Tel:6682164;Fax:6672989.

beatrizferro@cideim.org.co

Recibido: 28/12/05; aceptado: 22/04/06
}

En Colombia la leptospirosis ha sido estudiada principalmente desde el punto de vista de la salud animal, siendo una enfermedad prevalente en el ganado vacuno y porcino, entre otros (9-11). También se han realizado estudios de prevalencia en personas expuestas por riesgo ocupacional, encontrándose anticuerpos anti-Leptospira entre el 13,1 y el $22,4 \%$ de las personas evaluadas $(9,12)$; sin embargo, poco se conoce acerca del comportamiento de la leptospirosis en entornos urbanos.

El objetivo de este estudio fue determinar la seroprevalencia de infección por Leptospira, identificar los serovares reactivos y explorar algunos factores asociados a seropositividad en barrios periféricos de Cali. La información generada contribuirá al conocimiento de esta zoonosis en nuestro medio.

\section{Materiales y Métodos}

\section{Área de estudio}

Se realizó un estudio transversal de prevalencia de infección en habitantes de tres barrios periféricos de la ciudad de Cali, que fueron seleccionados por presentar condiciones favorables para la transmisión de leptospirosis, tales como la existencia de calles sin pavimentar, cercanía a basureros y canales de aguas lentas, además de facilidad de acceso. Los barrios seleccionados fueron: Petecuy I-III (Jarillón Vegas), Puerto Nuevo y Rodrigo Lara Bonilla, localizados en las comunas 6,7 y 13 , respectivamente. Este estudio fue aprobado por el Comité Institucional de Ética de Investigación en Humanos del CIDEIM y se realizó en el periodo de enero a julio de 2004.

\section{Tamaño de muestra y muestreo}

Para estimar la prevalencia de infección por Leptospira se calculó un tamaño de muestra de 255 individuos, teniendo en cuenta una prevalencia esperada del $20 \%$ (13), un nivel de confianza del 95\% y un error de estimación del $5 \%$.

El muestreo fue estratificado y proporcional al número de habitantes por barrio, sexo y edad. Para la asignación de cuotas por sexo y rangos de edad (< 14 años, 15 a 35 años, 36 a 56 años y 57 años o más) se tuvieron en cuenta datos de la 
alcaldía de Cali (14) y datos censales provistos por los líderes de cada barrio sobre el número aproximado de habitantes.

El muestreo comprendió todas las manzanas en cada barrio, y con base en un croquis previamente elaborado por líderes comunitarios se seleccionaron las viviendas de manera sistemática, casa de por medio, desde la vivienda inicial del muestreo escogida arbitrariamente. Sólo una persona de cada vivienda, seleccionada al azar entre los voluntarios mediante la generación de números aleatorios por calculadora, fue invitada a participar en el estudio. Después de explicar claramente los objetivos, procedimientos, posibles riesgos y beneficios, se solicitó la autorización de cada persona mediante la firma del consentimiento informado. Los datos epidemiológicos como edad, sexo, localización, así como el tiempo de residencia en el mismo barrio, ocupación y exposición a factores potencialmente asociados con leptospirosis, como fuentes de agua o contacto con animales domésticos (perros, cerdos, etc.) o peridomésticos (roedores), se recogieron a través de una encuesta. Una vez completada la cuota necesaria para un grupo de sexo y edad determinada en cada barrio, éste se cerraba y el muestreo continuaba para los otros grupos hasta completar el tamaño de muestra requerido.

\section{Prueba de microaglutinación (MAT)}

De cada persona se tomó una muestra de $5 \mathrm{~mL}$ de sangre por venopunción en la fosa antecubital del brazo no dominante del paciente. Las muestras fueron tomadas en tubo Vacutainer ${ }^{\circledR}$ sin anticoagulante, se dejaron coagular y el suero resultante fue congelado a $-70^{\circ} \mathrm{C}$ hasta su uso. Para la determinación de la seroprevalencia se evaluó la reactividad de los anticuerpos contra Leptospira por medio de la prueba de MAT, usando la metodología convencional (15) con un panel de 19 serovares de Leptospira como antígenos vivos. Estas cepas pertenecían a 16 serogrupos: Australis (serovar Australis), Autumnalis (serovar Autumnalis), Bataviae (serovar Bataviae), Canicola (serovar Canicola), Ballum (serovar Castellonis), Cynopteri (serovar Cynopteri), Grippothyphosa (serovar Grippothyphosa),
Hebdomadis (serovar Hebdomadis), Icterohaemorrhagiae (serovar Icterohaemorrhagiae, Copenhageni), Javanica (serovar Javanica), Panama (serovar Panama), Seramanga (serovar Patoc), Pomona (serovar Pomona), Pyrogenes (serovar Pyrogenes), Sejroe (serovares Sejroe, Wolffi, Hardjo) y Tarassovi (serovar Tarassovi). Los serovares incluidos fueron proporcionados por Albert Ko de la Fundación Instituto Oswaldo Cruz, FIOCRUZ, Salvador, Bahía, Brasil. Las leptospiras usadas como antígenos fueron cultivadas en medio líquido Ellinghausen-McCullough-JohnsonHarris (Difco), y entre el día 4 y 10 de crecimiento se ajustaron a un estándar de McFarland de 0,5. La prueba se realizó en microplacas de 96 pozos agregando $50 \mu \mathrm{L}$ de cada antígeno a $50 \mu \mathrm{L}$ del suero previamente diluido a 1:25 con solución salina amortiguada con fosfatos. Las placas se cubrieron e incubaron durante dos horas a temperatura ambiente. La prueba se interpretó como positiva al observar por microscopia de campo oscuro y con objetivo de $10 \mathrm{X}$ una aglutinación igual o mayor al $50 \%$ de las leptospiras con al menos uno de los serovares y en una dilución del suero de 1:50. Los sueros positivos fueron titulados realizando diluciones seriadas dobles. Por cada corrida de la prueba se montó un control positivo, uno negativo y un control de antígenos. Adicionalmente, el grupo encargado del diagnóstico participó en la tercera y cuarta ronda de control de calidad externo para MAT, realizada por la Sociedad Internacional de Leptospirosis, obteniéndose resultados concordantes que permitieron garantizar la identidad antigénica de los serovares utilizados.

\section{Análisis estadístico}

La información se ingresó en una base de datos diseñada en Access 2000 y el análisis de los datos se realizó con el paquete estadístico SPSS 7.5 para Windows (SPSS Inc., Chicago, 1996). Se realizó un análisis descriptivo de las variables categóricas en estudio, expresando frecuencias absolutas y relativas. Para las variables numéricas se utilizaron medidas de tendencia central y dispersión.

Sólo las variables con respuesta fueron incluidas en el análisis. La prevalencia de infección por 
Leptospira se expresó con su respectivo intervalo de confianza del $95 \%$, ajustada de acuerdo al diseño del muestreo. Se evaluó la asociación entre la presencia de anticuerpos y los factores potencialmente asociados con leptospirosis, usando la prueba de ji cuadrado o la prueba exacta de Fisher según el caso, y considerando un $p<0,05$ como significativo.

\section{Resultados}

Se obtuvo un total de 259 muestras de suero de voluntarios, 63 de Petecuy I-III (Jarillón Vegas), 96 de Puerto Nuevo y 100 de Rodrigo Lara Bonilla, de las cuales 59 (23,3\% [IC95\% 18,3 a 28,3]) fueron positivas para al menos un serovar. La frecuencia de anticuerpos fue superior al $15 \%$ en todos los grupos de edad, principalmente en los sujetos mayores de 57 años, aunque no se encontraron diferencias significativas entre ellos (cuadro 1). La prevalencia encontrada fue significativamente mayor en hombres que en mujeres (59,3\% vs. 40,7\%, $p=0,045)$ (cuadro 1).

La población evaluada en los tres barrios del estudio correspondió principalmente a estudiantes $(37,1 \%)$, amas de casa (32\%), trabajadores en oficios varios $(24,6 \%)$ y otros $(6,3 \%)$. La mediana del tiempo de permanencia en los barrios fue de 12 años (rango: 1 mes a 56 años). En cuanto a los factores potencialmente asociados con la presencia de anticuerpos anti-Leptospira explorados en la encuesta (cuadro 2), un bajo porcentaje de personas, 24/259 (9,3\%), manifestó realizar alguna actividad en el río, laguna u otra fuente de agua. Por el contrario, el caminar descalzo dentro y fuera de la vivienda fue reportado por el 70,9 y el $44,7 \%$ de la población, respectivamente. Respecto al contacto con roedores, una alta proporción de las personas encuestadas reportó haber visto ratas y/o ratones dentro $(91,5 \%)$ o fuera de la vivienda (89,5\%). La convivencia de la población encuestada con los animales domésticos fue del $72,5 \%$, principalmente perros, gatos, cerdos, conejos, caballos y vacas. Se encontró una asociación estadísticamente significativa entre el contacto con animales $(p=$ $0,038)$, en particular con cerdos $(p=0,042)$ y conejos $(p=0,049)$ y la presencia de anticuerpos anti-Leptospira. Ninguno de los otros factores
Cuadro 1. Serorreactividad a Leptospira por edad y sexo en la población estudiada de tres barrios periféricos de Cali.

\begin{tabular}{lcc}
\hline & $\begin{array}{c}\text { Positivos } \\
\mathbf{n}(\%)\end{array}$ & $\begin{array}{c}\text { Total } \\
\mathbf{n}\end{array}$ \\
\hline $\begin{array}{l}\text { Grupos de Edad } \\
\text { (años) }\end{array}$ & & \\
14 o menos & $13(15,9)$ & 82 \\
$15-35$ & $25(27,8)$ & 90 \\
$36-56$ & $12(19,4)$ & 62 \\
57 o más & $9(36,0)$ & 25 \\
Sexo* & & \\
Masculino & $35(59,3)$ & 124 \\
Femenino & $24(40,7)$ & 135 \\
Total & $59(23,3)$ & 259 \\
\hline
\end{tabular}

${ }^{*} p=0,045$

mencionados presentó asociación estadística significativa, a pesar de que el contacto con gatos mostró un $p=0,064$ (cuadro 2).

Del panel de 19 serovares evaluados, se encontró reactividad frente a 16 que representaban 14 de los 16 serogrupos incluidos. No se detectaron anticuerpos contra tres serovares: Javanica, Hardjo y Tarassovi (figura 1). Los serovares frente a los cuales hubo mayor reactividad fueron Grippothyphosa (10,4\%), Patoc (8,5\%), Copenhageni (2,3\%), Panama (2,3\%) y Pomona $(2,3 \%)$; en general los títulos de anticuerpos fueron bajos (rango: 1:50 a 1:1600).

\section{Discusión}

En este estudio se encontró una prevalencia de leptospirosis de $23,3 \%$ en muestras de suero de voluntarios de tres barrios periféricos de Cali, lo cual evidencia la transmisión urbana de esta zoonosis en la ciudad. La mayoría de los estudios de prevalencia de leptospirosis en Colombia se han realizado en animales de producción y en personas expuestas por su ocupación (9-12). Sin embargo, los datos encontrados en este estudio sugieren que la transmisión de leptospirosis no sólo está ocurriendo en las situaciones tradicionalmente descritas, sino también en el entorno urbano, lo cual ha sido informado en algunas ciudades de países como Brasil, Perú, Cuba, Nicaragua, México y Estados Unidos, entre otros $(2-4,6-8,16,17)$, donde se han encontrado seroprevalencias de 14,2\% (Estado de Yucatán, 
Cuadro 2. Factores potencialmente asociados con la presencia de anticuerpos anti-Leptospira en la población estudiada de tres barrios periféricos de Cali.

\begin{tabular}{|c|c|c|c|c|c|}
\hline Variable & $\begin{array}{l}\text { Positivos } \\
\text { n (\%) }\end{array}$ & $\mathbf{n}$ & $\begin{array}{l}\text { Negativos } \\
\text { n (\%) }\end{array}$ & $\mathbf{n}$ & Valor $p^{\mathrm{a}, \mathrm{b}}$ \\
\hline \multicolumn{6}{|l|}{$\begin{array}{l}\text { Realizar alguna actividad en el río, } \\
\text { laguna u otra fuente de agua: }\end{array}$} \\
\hline bañarse & $7(11,9)$ & 59 & $14(7,1)$ & 197 & $0,279^{b}$ \\
\hline pescar & $4(6,8)$ & 59 & $6(3,0)$ & 197 & $0,245^{b}$ \\
\hline lavar ropa & $1(1,7)$ & 59 & $3(1,5)$ & 197 & $1,000^{b}$ \\
\hline \multicolumn{6}{|l|}{ Caminar descalzo } \\
\hline dentro de la vivienda & $40(67,8)$ & 59 & $143(71,9)$ & 199 & $0,546^{a}$ \\
\hline fuera de la vivienda & $29(50,0)$ & 58 & $86(43,2)$ & 199 & $0,361^{a}$ \\
\hline \multicolumn{6}{|l|}{ Presencia de ratas o ratones } \\
\hline dentro de la vivienda & $55(93,2)$ & 59 & $182(91,0)$ & 200 & $0,591^{a}$ \\
\hline fuera de la vivienda & $52(89,7)$ & 58 & $178(89,4)$ & 199 & $0,964^{\mathrm{a}}$ \\
\hline Contacto con animales domésticos & $49(83,1)$ & 59 & $138(69,3)$ & 199 & $0,038^{a}$ \\
\hline \multicolumn{6}{|l|}{ Animales domésticos en la vivienda: } \\
\hline perros & $39(66,1)$ & 59 & $112(56,0)$ & 200 & $0,167^{a}$ \\
\hline gatos & $23(39,0)$ & 59 & $53(26,5)$ & 200 & $0,064^{a}$ \\
\hline cerdos & $16(27,1)$ & 59 & $31(15,5)$ & 200 & $0,042^{a}$ \\
\hline conejos & $7(12,1)$ & 58 & $8(4,0)$ & 199 & $0,049^{b}$ \\
\hline caballos & $2(3,4)$ & 59 & $6(3,0)$ & 200 & $1,000^{b}$ \\
\hline vacas & $0(0,0)$ & 58 & $2(1,0)$ & 200 & $1,000^{b}$ \\
\hline
\end{tabular}

a. Prueba de ji cuadrado

b. Prueba exacta de Fisher

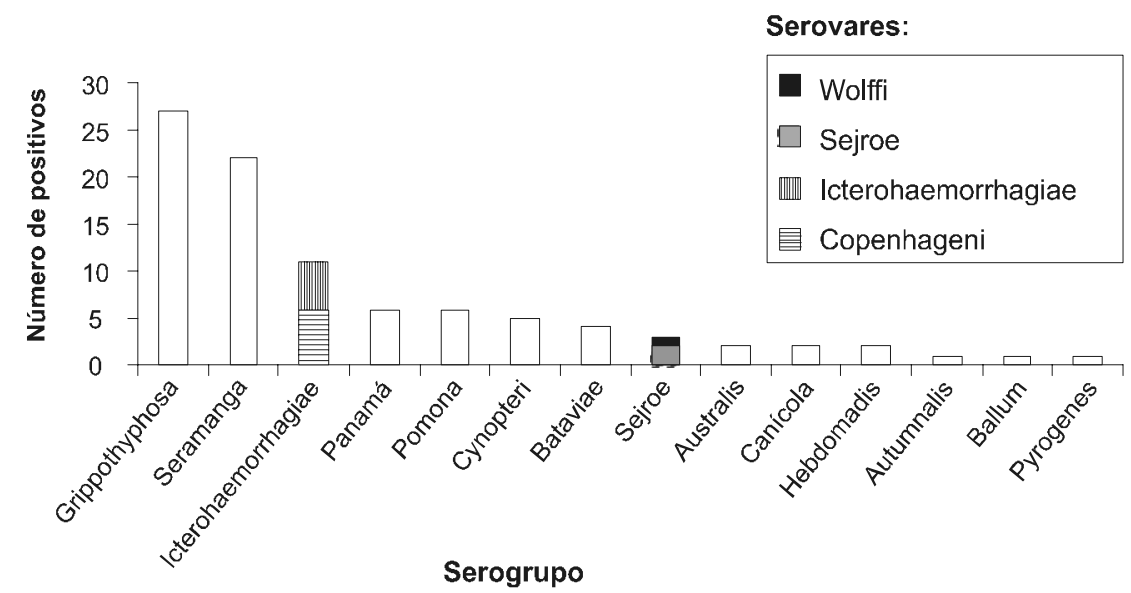

Figura 1. Distribución de la reactividad para Leptospira por serogrupo en barrios periféricos de Cali.

México) (17), 16\% (Baltimore, EE.UU.) (4), y $28 \%$ (Iquitos, Perú) (6).

En este estudio se encontró una diferencia significativa en la prevalencia de infección entre mujeres y hombres, siendo mayor en estos últimos, hallazgo que coincide con lo reportado previamente en otros estudios en Latinoamérica
$(7,17)$. De igual manera, la presencia de anticuerpos fue más alta en el grupo de adultos mayores $y$, aunque este hallazgo no fue estadísticamente significativo, es similar a lo encontrado en otras regiones $(6,7,17)$, y ha sido explicado por la hipótesis de que la exposición continua a través de la vida puede resultar en un 
incremento edad dependiente de la seropositividad de anticuerpos (6).

A pesar de no ser un estudio diseñado para la identificación de factores de riesgo, se exploraron algunos factores previamente relacionados con la presencia de anticuerpos para Leptospira $(3,6,16,17)$. Solamente se encontró asociación estadísticamente significativa con el contacto con animales, en particular cerdos y conejos.

Los perros se han considerado como un factor importante para la transmisión de leptospiras en ambientes urbanos (7), y se ha evidenciado su posible papel por la presencia de anticuerpos antiLeptospira en estos animales (18). Sin embargo, en el presente estudio no se encontró asociación entre la presencia de anticuerpos en los humanos y el contacto con los perros, que fueron los animales domésticos con los que tuvo más contacto la población encuestada.

Algunos estudios realizados en Latinoamérica, han evidenciado factores de riesgo para leptospirosis. En un estudio similar en México, se encontró asociación significativa con la presencia de roedores y el contacto con fuentes naturales de agua (17). Por otro lado, en un estudio desarrollado en Nicaragua para evaluar factores de riesgo, se encontró asociación con vivir en zonas rurales, lavar ropa o bañarse en un río o arroyo, y desarrollar algunas actividades ocupacionales, pero no se observó asociación con la exposición a animales domésticos ni a roedores (16). En Perú también se identificaron como factores de riesgo bañarse o vivir cerca del río, vivir en áreas con pobres condiciones sanitarias y no usar zapatos, pero no se encontró asociación con la presencia de animales en la casa o el trabajo (6).

De otra parte, en este estudio se empleó una batería de 19 serovares, representantes de los 16 serogrupos recomendados por la ILS para identificar infecciones por serovares desconocidos a través de la prueba de MAT (19), lo que permite evaluar la reactividad frente a un grupo amplio de leptospiras. Se observó aglutinación con 14 de los 16 serogrupos incluidos, siendo los más frecuentes Gryppotyphosa, Seramanga, Icterohaemorrhagiae, Panama, Pomona,
Cynopteri, Bataviae, Sejroe, Australis y Canicola. En este aspecto se encuentran algunas diferencias entre lo informado en otras regiones como México, donde los principales serogrupos a los que se dirigió la respuesta serológica evaluada fueron Tarassovi, Sejroe, Pomona y Panama (17), y Brasil, en donde se ha encontrado que la respuesta serológica está dirigida casi exclusivamente al serogrupo Icterohaemorrhagiae $(2,3)$.

La información sobre frecuencia de anticuerpos frente a los serogrupos permitirá redefinir el panel de los serovares necesario para realizar la prueba de MAT de acuerdo a la reactividad observada localmente; no obstante, es importante resaltar que sería ideal incluir aislamientos locales para mejorar el desempeño de la prueba en la identificación de infecciones por leptospiras.

En este estudio, los anticuerpos encontrados estaban dirigidos a varios serogrupos y sus serovares, los que a su vez han sido asociados con diferentes reservorios. Tal es el caso del serovar Gryppotyphosa, que ha sido asociado con mapaches, marsupiales y bovinos, los serovares Icterohaemorrhagiae y Copenhageni, con ratas, y el serovar Pomona, con ganado porcino y bovino $(1,5)$. Sin embargo, también se encontró que un $8,5 \%$ de los sueros tenía anticuerpos frente al serovar Patoc, el cual se considera saprofito. Esta diversidad en la reactividad serológica podría explicarse por el alto grado de reacciones cruzadas que se presentan entre los distintos serogrupos y sus serovares, debido en parte a antígenos comunes entre las leptospiras, o por anticuerpos que persisten de contactos infecciosos previos con diferentes leptospiras (20), lo cual puede generar reacciones paradójicas que hacen difícil interpretar las pruebas serológicas como el MAT (5). En cualquier caso, la utilidad del MAT en este estudio es la de detectar la presencia de anticuerpos y dar una impresión general acerca de los serogrupos circulantes en la población evaluada. Sin embargo, no podría descartarse el hecho de que en estas zonas periféricas de Cali, diferentes reservorios, que a su vez portan diferentes serovares, y diferentes fuentes naturales sean las responsables del mantenimiento y transmisión de leptospiras, a 
pesar de que se ha establecido que los principales reservorios urbanos son roedores y caninos $(1,7)$.

Los resultados obtenidos en este estudio representan un aporte a la línea de base del conocimiento sobre la prevalencia y transmisión de leptospiras en ambientes urbanos en Colombia, en particular en áreas periféricas de la ciudad de Cali. Se requieren futuros estudios sobre la transmisión y los factores de riesgo, así como el establecimiento de un sistema de vigilancia, para un mejor entendimiento de esta zoonosis en nuestro medio y para la adecuada formulación de medidas de prevención y control.

\section{Agradecimientos}

Los autores del presente artículo agradecen a Albert Ko por su asesoría y donación de las cepas de referencia para realizar los estudios serológicos y a la Dra. Raydel Martínez por sus sugerencias para el diseño del estudio. Igualmente agradecen al Centro de Zoonosis y Unidad Ejecutoria de Saneamiento, entidades dependientes de la Secretaria de Salud Pública Municipal, y a Martha Liliana López por su valioso apoyo logístico y trabajo técnico en el campo. A los líderes comunitarios y comunidad en general de los barrios Petecuy I-III (Sector del Jarillón), Puerto Nuevo y Rodrigo Lara Bonilla por la colaboración prestada, y a Mauricio Santafé, Angélica Aponte, Carlos Quiceno y Diana Isabel Lizcano por su apoyo profesional.

\section{Financiación}

Colciencias: Proyecto 2229-04-12616, Contrato No. 095-2003, CIDEIM.

\section{Conflicto de intereses}

Los autores manifestamos que no existe ningún conflicto de intereses sobre los resultados presentados en este artículo.

\section{Referencias}

1. Barthi AR, Nally JE, Ricaldi JN, Matthias MA, Díaz MM, Lovett MAm, et al. Leptospirosis: a zoonotic disease of global importance. Lancet Infect Dis 2003;3:757-71.

2. Romero EC, Bernardo CC, Yasuda PH. Human leptospirosis: a twenty-nine-year serological study in
Sao Paulo, Brazil. Rev Inst Med Trop Sao Paulo 2003;45:245-8.

3. Ko Al, Galvao Reis M, Ribeiro Dourado CM, Jonson WD Jr, Riley LW. Urban epidemic of severe leptospirosis in Brazil. Salvador Leptospirosis Study Group. Lancet 1999;354:820-5.

4. Vinetz JM, Glass GE, Flexner CE, Mueller P, Kaslow DC. Sporadic urban leptospirosis. Ann Intern Med 1996;125:794-8.

5. Levett PN. Leptospirosis. Clin Microbiol Rev 2001;14:296-326.

6. Johnson MA, Smith H, Joeph P, Gilman RH, Bautista CT, Campos KJ, et al. Environmental exposure and leptospirosis, Peru. Emerg Infect Dis 2004;20:1016-22.

7. Lomar AV, Diament D, Torres JR. Leptospirosis in Latin America. Infect Dis Clin North Am 2000;14:23-39.

8. Barcellos C, Sabroza PC. Socio-environmental determinants of the leptospirosis outbreak of 1996 in the western Rio de Janeiro: a geographical approach. Int J Environ Health Res 2000;10:301-13.

9. Ochoa JE, Sánchez A, Ruiz I. Epidemiología de la leptospirosis en una zona andina de producción pecuaria. Rev Panam Salud Publica 2000;7:325-31.

10. Orrego-Uribe A, Giraldo de León G, Ríos-Arango B, Valencia-Prada PA. Leptospirosis en personas de riesgo de quince explotaciones porcinas y de la central de sacrificio de Manizales, Colombia. Arch Med Vet 2003;35:205-13.

11. Griffiths IB, Gallego MI, De Leon LS. Levels of some reproductive diseases in the dairy cattle of Colombia. Trop Anim Health Prod 1984;16:219-23.

12. Najera S, Alvis N, Babilonia D, Alvarez L, Mattar S. Leptospirosis ocupacional en una región del caribe colombiano. Salud Pública Mex 2005;47:240-4.

13. Ministerio de Salud. Sistema Nacional de Vigilancia en Salud Pública. Informe Ejecutivo Semanal. Oficina de Epidemiología. Semana Epidemiológica SIVIGILA No. 13. Situación actual de la leptospirosis en el país. Marzo 26 a abril 1 de 2000 .

14. Departamento Administrativo de Planeación Municipal. Actualización de la base demográfica de Cali por comuna y barrio ajustado al Censo de 1993. Consultor: Juver Galeano Loaiza. Documento final. Santiago de Cali: Departamento Administrativo de Planeación Municipal; 1997.

15. Myers DM. Leptospirosis. Manual de métodos para el diagnóstico de laboratorio (Nota técnica No 30). Buenos Aires: Centro Panamericano de Zoonosis, OPS/OMS; 1985.

16. Ashford DA, Kaiser RM, Spiegel RA, Perkins BA, Weyant RS, Bragg SL, et al. Asymptomatic infection 
and risk factors for leptospirosis in Nicaragua. Am J Trop Med Hyg 2000;63:249-54

17. Vado-Solis I, Cárdenas-Marrufo MF, JiménezDelgadillo B, Alzina-López A, Laviada-Molina H, Suárez-Solis $\mathrm{V}$, et al. Clinical-epidemiological study of leptospirosis in humans and reservoirs in Yucatán, México. Rev Inst Med Trop Sao Paulo 2002;44:335-40.

18. Rodríguez AL, Ferro BE, Varona MX, Santafé M. Evidencia de exposición previa a Leptospira en perros callejeros de Cali. Biomédica 2004;24:291-5.
19. Terpstra WJ. Human leptospirosis: guidance for diagnosis, surveillance and control. Malta: International Leptospirosis Society, World Health Organization; 2003.

20. Tansuphasiri U, Deepradit S, Phulsuksombati D, Tangkanakul W. A test strip IgM Dot-ELISA Assay using leptospiral antigen of endemic strains for serodiagnosis of acute leptospirosis. J Med Assoc Thai 2005;88:391-8.

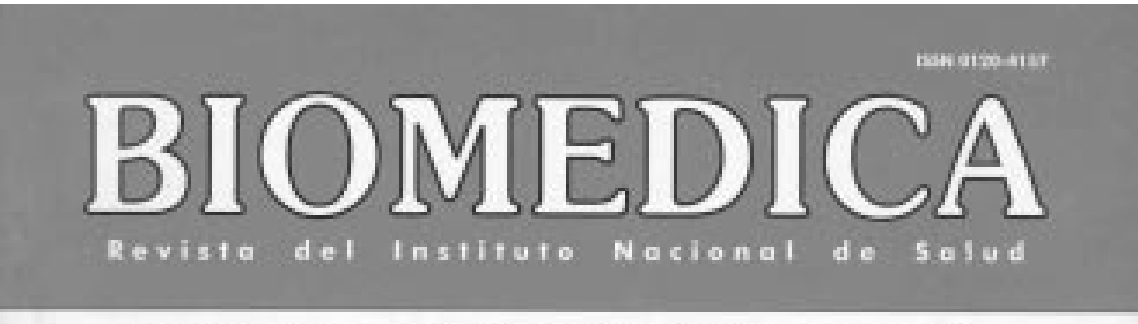

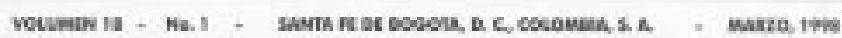

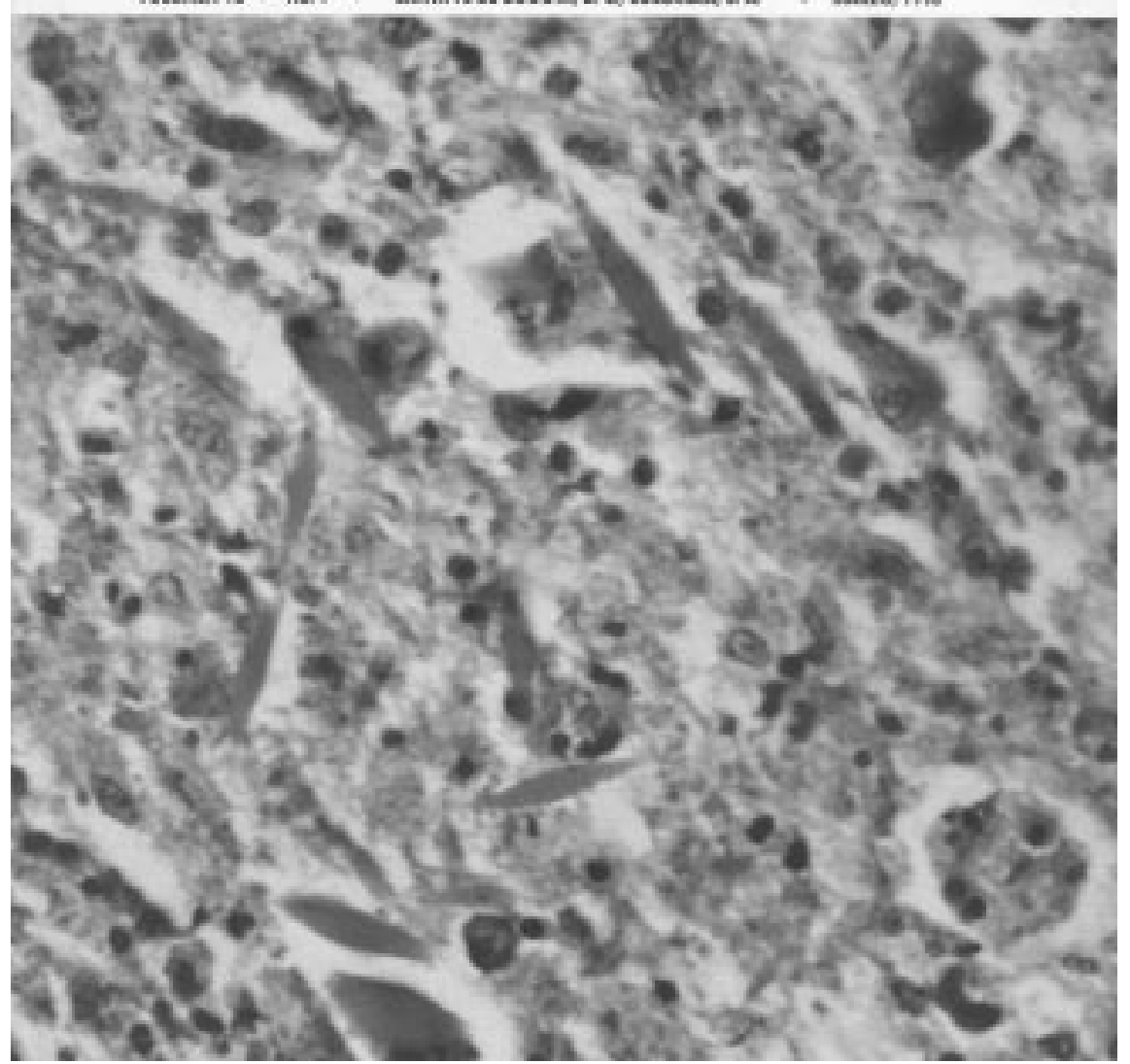

